\title{
Analysis of maximum transmissible power in electrified railway system
}

\author{
Etienne Sourdille ${ }^{1}$, Florence Ossart ${ }^{2}$, Jean Bigeon ${ }^{3}$, Alexandre Machet ${ }^{1}$ \\ ${ }^{1}$ SNCF, Direction de l'Ingénierie, 6 avenue François Mitterrand, 95574 La Plaine St-Denis \\ ${ }^{2}$ GEEPS - CNRS UMR 8507 - CentraleSupélec - UPSud - Sorbonne Université UPMC; 91192 Gif sur Yvette - France \\ ${ }^{3}$ G-SCOP - CNRS UMR 5272; Grenoble INP-UJF; 38000 Grenoble - France
}

\begin{abstract}
The maximum transmissible electrical power concept is applied to different electrical railway systems. Different problems arise depending on whether the electrical system is using DC or AC system. A DC example illustrates how the power line loadability can be assessed. Afterwards, the definition is extended to $\mathrm{AC}$ system to take into account the effect of the reactive power. Finally, an $\mathrm{AC}$ use case with an high voltage booster shows the importance of these phenomena and some difficulties to properly simulate a system close to its limit.
\end{abstract}

Keywords - Electric railway modeling; railway application; Transmissible power; Reactive power compensation

\section{INTRODUCTION}

The growth of traffic on existing or newly electrified railway requires sizing study for power supply facilities in order to meet standard needs and expected performances. This type of study is conducted using railway simulators [1-4]. In specific cases, the existing power supply facilities cannot be reinforced (unusually high traffic, or difficult access) and the system is operated close to its physical limits. The limiting concepts of maximum transmissible power and voltage collapse must then be well understood and handled for correct simulation.

The present paper recalls these concepts and reports how they are accounted for: simulation with given power loads leads to a nonlinear problem, and the residue of the non-linear calculation is used as an indicator to detect when physical limits are exceeded and voltage instability takes place. This approach is used in a particular AC system use case, in which a high voltage booster (HVB) is used to control the voltage.

\section{PRINCIPLE OF TRANSMISSIBLE POWER}

The concept of maximum transmissible power is fairly well known and characterized for electrical power system. It enables to characterize the loadability of the system as well as its voltage stability given admissible boundaries for the voltages. For a railway electrical system, it is harder to define a link between the transmissible power and an effective acceptable load. Indeed the trains are moving along the feeding lines, and their load can vary rapidly. A train can stop and accelerate suddenly, thus either disappearing or presenting a full load depending on its state. The mobile nature of the train also means that the load location is always changing. This complicates the determination of a maximum load along the line.
Unlike usual electrical power feeding system, railway system standards allow much larger differences between the minimum and maximum admissible voltages: this difference can reach up to $50 \%$ of the nominal voltage. It should also be noted that the lowest admissible voltage is close to the voltage corresponding to the maximum transmissible power of the line, meaning that the system is operated close to its stability point. This requires precise sizing, based on accurate and robust simulation models as it will be exposed later.

Conversely, when the trains use regenerative braking, they behave like power generators and locally rise the line voltage. Upper limits may then have to be considered. This situation is met for recent rolling stock using four quadrant converters. In that case, the standard [8] defines the upper voltage limit.

\section{A. Railway network overview}

A variety of electrical systems coexist in Europe using either direct-current (DC) or alternating-current (AC). Nonetheless, some common elements can be defined. A railway electrical network is composed of different facilities in order to supply electrical power to the train:

- Sources: feeding substations including transformers, rectifiers, etc...

- Connecting elements: contact line system (CLS) including contact wire and messenger wire, rail, paralleling post, auto-transformer...

- Loads: trains and traffic.

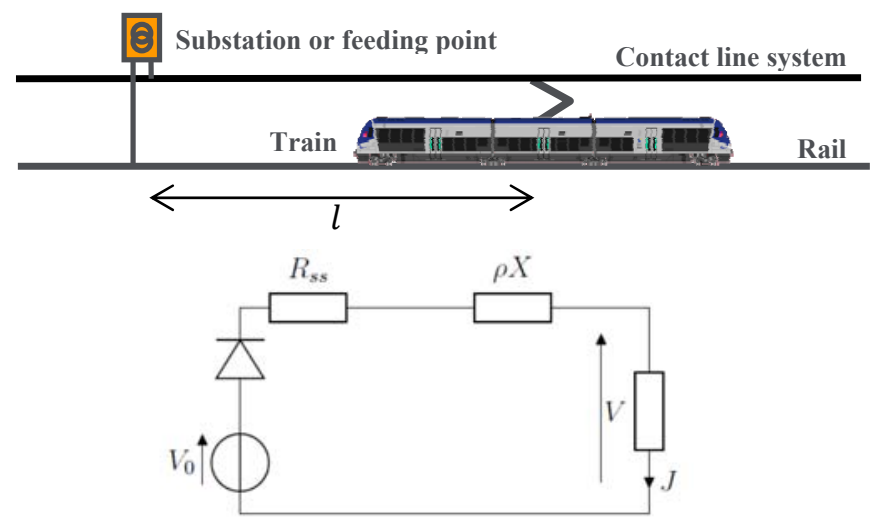

Fig. 1 : Simplified model of a DC electrical railway system with a single train on a single track 
Fig 1. provides a simplified description of a railway DC electrical system with a single train on a single track. The different variables represent:

- $V_{0}$ : open-circuit voltage of the feeding substation.

- $R_{S S}$ : equivalent resistance of the feeding substation, including the rectifier.

- $\rho$ : linear resistance of the CLS for one track.

- $d$ : distance between the train and the feeding substation.

- $J$ : current absorbed by the train.

- $\quad V$ : pantograph voltage.

In railway electrical system, European standards [7], [8] allow the pantograph voltage to vary in a large range. For example, in $1500 \mathrm{~V}$ DC system, the minimum and maximum voltage are respectively $1000 \mathrm{~V}$ and the $1950 \mathrm{~V}$. Thus the difference is $950 \mathrm{~V}$, which represents $54 \%$ of the nominal voltage. In high load cases, the system can thus be close to its voltage stability level (875 V).

As a side note, the standard [7] also specifies that the maximum load current is limited for the train. For the sake of simplicity, this will not be considered here as it introduces a feedback loop into the loadability. Indeed, this defines an interaction between the train current limit and the voltage level.

\section{B. Maximum transmissible power : DC case}

Two physical limits naturally emerge to assess the loadability of an electrical network: the current and the load voltage.

The current is mostly limited by thermal constraints on the different elements on the system. For example, the temperature of the rail must be limited to avoid excessive dilatation. Thermal constraints are often expressed in term of heating and require the knowledge of the entire history of the load signal. As such, it can be difficult to translate into admissible power limit or distribution and falls beyond the scope of this article.

Concerning the maximum loadability problem in terms of voltage limit, we use the simple case defined in 0 as an illustration for a DC system. Regenerative breaking is not considered, so the diode is always on and the pantograph voltage $V$ is expressed as:

$$
V=V_{0}-\left(R_{S S}+\rho d\right) . J .
$$

The total resistance is denoted $R_{d}=R_{S S}+\rho d$ and the supplied power $P$ is expressed as a function of $V$ by:

$$
P=V . J=P=\frac{V \cdot\left(V_{0}-V\right)}{R_{d}} .
$$

This power is zero either for $V=V_{0}$ (open circuit) or $V=$ 0 (short circuit) and reaches a maximum for $V=\frac{1}{2} V_{0}$, as it can be seen on Fig. 2. The corresponding value is:

$$
P_{\max }=\frac{V_{0}^{2}}{4 R_{l}}
$$

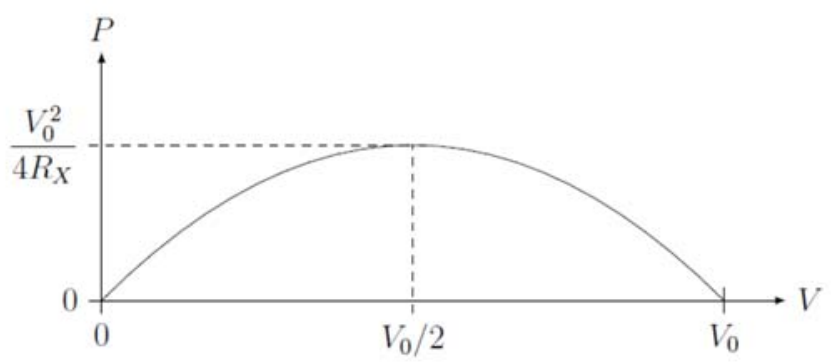

Fig. 2 : Train power as a function of the pantograph voltage.

$P_{\text {max }}$ is the maximal power that can be transmitted by the feeding system to the load, also called maximal transmissible power. This power can be expressed as a function of the shortcircuit power $P_{0}$ :

$$
P_{\max }=\frac{1}{4} P_{0} \quad \text { with } P_{0}=\frac{V_{0}^{2}}{R_{d}}
$$

This data is especially useful as we frequently exploit the system close to its limit: for the $1500 \mathrm{~V}$ DC system, standards state that the pantograph voltage lower limit is $1000 \mathrm{~V}$, whereas the stability limit is at $975 \mathrm{~V}$. The power at $1000 \mathrm{~V}$ represents $80 \%$ of the maximal transmissible power.

\section{Maximum transmissible power : AC case}

Fig. 1 applies to AC systems, provided that the diode is removed, resistances are replaced by impedances, and complex quantities are used. Let us denote:

- $\quad V_{0}$ : open-circuit voltage of the feeding substation, used as phase reference.

- $Z_{S S}$ : equivalent impedance of the feeding substation.

- $z$ : linear line impedance of the CLS for one track.

- $\underline{Z}=Z_{S S}+z d=R+j X$ : total impedance

- $\quad \underline{V}$ : pantograph complex voltage

- $J$ : complex current absorbed by the train

- $\underline{S}=P+j Q$ : complex apparent power of the load.

The complex apparent power $\underline{S}$ is defined by:

$$
\underline{S}=\underline{V} \cdot J^{*}
$$

Hence, (1) becomes :

$$
\underline{V}=V_{0}-\underline{Z} \cdot\left(\frac{\underline{S}}{\underline{V}}\right)^{*}
$$

Multiplying (6) by its conjugate and rearranging things lead to the second order real equation (7), in which the unknown is $U=|\underline{V}|^{2}$. Solutions exist if and only if the discriminant $\Delta$ defined by (8) is positive or zero.

$$
U^{2}+2 \cdot\left[\operatorname{Re}\left(\underline{Z} \cdot \underline{S}^{*}\right)-\frac{V_{0}^{2}}{2}\right] \cdot U+\left|\underline{Z} \cdot \underline{S}^{*}\right|^{2}=0 \text {. }
$$




$$
\Delta=\left[\operatorname{Re}\left(\underline{Z} \cdot \underline{S}^{*}\right)-\frac{V_{0}{ }^{2}}{2}\right]^{2}-\left|\underline{Z} \cdot \underline{S}^{*}\right|^{2}
$$

Using the parameter $t$ and $P_{m}$ defined hereunder, and which characterize the line and feeding substation, the condition $\Delta \geq 0$ is rewritten into (11).

$$
\begin{gathered}
t=\frac{X}{R}, \\
P_{m}=\frac{V_{0}{ }^{2}}{2 R} . \\
(t P-Q)^{2}+2(P+t Q) \cdot P_{m}-P_{m}{ }^{2} \leq 0 .
\end{gathered}
$$

Equation (11) defines the set of complex power that can actually be transmitted by the feeding system to the train. This set of points is delimited in the complex plane $(P, Q)$ by a parabola with a tilted axis $Q=t . P$. For a load with given complex power $\underline{S}$ within the area where $\Delta \geq 0$, (7) has two solutions given by (12). Both solutions are physically admissible, but the system actual operating point corresponds to the one with the "plus" sign.

$$
|\underline{V}|=\sqrt{-\left[\operatorname{Re}\left(\underline{Z} \cdot \underline{S}^{*}\right)-\frac{V_{0}^{2}}{2}\right] \mp \sqrt{\Delta}}
$$

One can also show [9] that operating points corresponding to a given load voltage $|\underline{V}|=V$ form a circle with parameters:

$$
\begin{gathered}
\text { center } C=\left(\beta^{2} R, \beta^{2} X\right), \\
\text { radius } r=\beta . V, \\
\text { where } \beta=\frac{V}{|Z|^{\circ}}
\end{gathered}
$$

Fig. 3 shows these elements for a given AC $25 \mathrm{kV}$ feeding system with a load at a given distance from the substation. The red parabola limits the transmissible power domain. The black lines represent loads with three different power factors: 0.8 inductive, 1 and 0.8 capacitive. The corresponding voltagepower curves are plotted on Fig. 4, where the blue and red parts correspond to the two solutions of (12). One can see the strong influence of the power factor on the maximum active power and the line voltage. A capacitive load is needed to compensate for the inductive reactive power of the feeding system and improve the power transmission. The train being an inductive load, capacitive compensation devices are required for that. For example, static VAR compensators (SVC) are basically capacitors whose capacitance is controlled according to some voltage constraints. European standards specify that the line voltage operating range is [19 $\mathrm{kV}-$ $27.5 \mathrm{kV}$ ], with peak values up to $29 \mathrm{kV}$. Hence, using the circle of given line voltage (blue lines in Fig. 3) is very helpful as it allows to define the complex power domain which respect these limits. These curves show that adding capacitive reactive power to the load enables to improve the transmissible active power and the line voltage, but only to some extent.

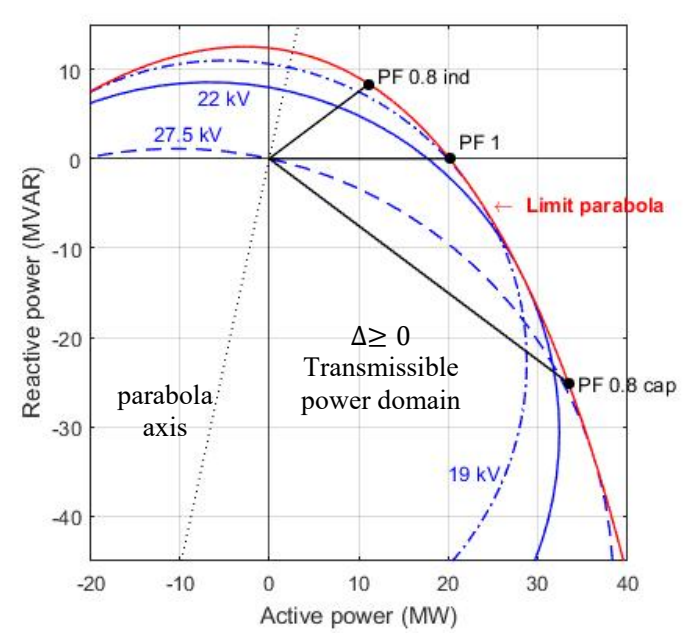

Fig. 3 : Transmissible power domain : the red parabola is the border of the domain; the blue circles correspond to given load voltages and the black lines to given load power factor .

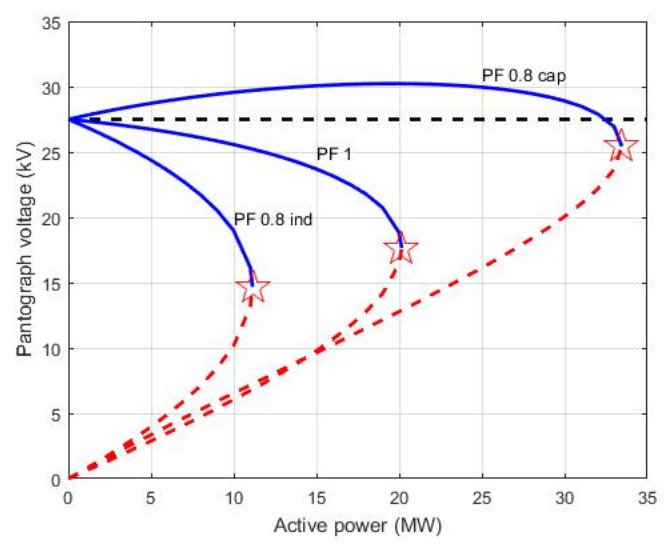

Fig. 4 : Pantograph voltage calculated using (12) for loads with three different power factors ( 0.8 inductive, $1,0.8$ capacitive).

The stars indicate the maximum transmissible power point.

\section{AC SYSTEM USE CASE}

The physical limit of maximum transmissible power and sustainable voltage had to be handled in a recent study with imposed $1 \times 25 \mathrm{kV}$ AC power supply equipments and an increasing traffic.

\section{A. System description}

The considered line runs through a $50 \mathrm{~km}$ long tunnel, which induces strong volume and cost design constraints. The system consists of a one track overhead line with a single feeding substation at one end and a static VAR compensators (SVC) at the other end. The supply system is distributed along the line, with isolated cables connecting the substation to intermediate feeding points about every $10 \mathrm{~km}$. This introduces parasitic capacitances which must be compensated, for example using self-inductances.

The line was originally designed and sized for a traffic of approximately 6 simultaneous trains. Following a demand to 
increase the traffic, the challenge has then been to upgrade the existing infrastructure without adding a new substation. Indeed, adding a new substation implies new electrical sections, which is not desirable because the train must stop their engines during the transition between the different sections, thus slowing their missions. Furthermore, a new substation requires a new contract with an energy supplier.

Because of the tunnel configuration, it is very difficult and expensive to add any equipment along the line. Therefore, moving toward a $2 \times 25 \mathrm{kV}$ system has been ruled out.

Another solution would be to add some feeders in parallel with the catenary. The feeders are un-insulated cables, made of copper or aluminum. The addition of parallel conductors lowers the line impedance, but inductive effects limit this decrease and the global impedance of the catenary system quickly reaches an asymptotic value when adding feeders, as shown by Fig. 5 .

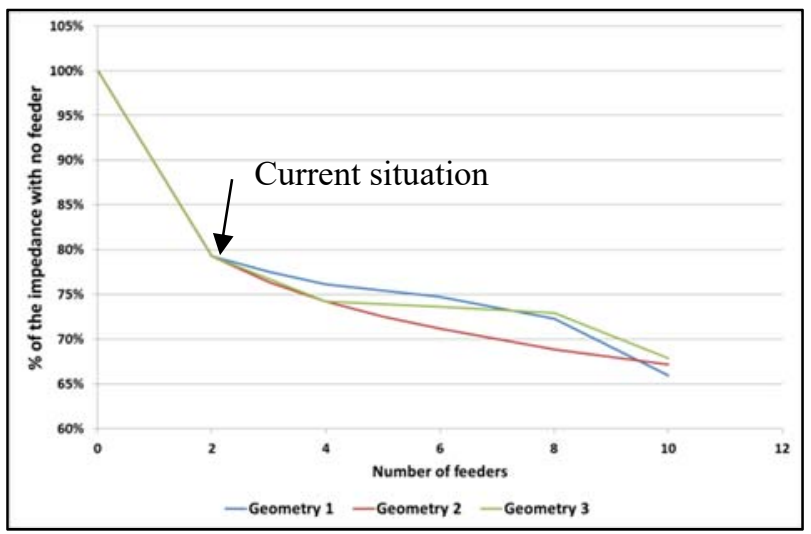

Fig. 5 : Line impedance versus number of additional feeders.

In the current situation, a $20 \%$ impedance reduction has already been obtained. An additional $10 \%$ gain would require
8 additional cables. Thus, this solution is not sustainable in terms of cost, setup complexity and volume inside the tunnel confined space. An alternative would be the use of a rigid conductor line which basically consists in an aluminum rail with a large cross section (comparable to 8 parallel feeders) using limited volume, and setup complexity. Again, due to the inductive effect, the gains are fairly limited compared to the current situation.

The last solution is to increase the static VAR compensator (SVC) capacity. The SVC basically consists in a capacitor bank with a capacitance that is adjusted in order to control the voltage at its terminals and keep it constant. When the maximum capacitance has been reached, the voltage can no longer be maintained at its set value. The device allows to improve the power factor of the system, thereby limiting ohmic losses and increasing the line transmittable power. Yet, as it was explained in section 2.B, this improvement has physical limits which must be well accounted for.

\section{B. Simulation and results}

In order to resize the SVC, simulations were carried out for a traffic of 8 simultaneous heavy trains. The speed profile and line voltage of the trains were calculated for increasing values of the SVC capacitance. For too small values of this capacitance, the power requested by the trains and voltage constraints are not fulfilled. The system behavior improves for higher values of the SVC capacitance. Fig. 6 shows an example of results : the pantograph voltage of one of the 8 trains is plotted as a function of its position on the line. The overall shape is correct, yet one notices local drops of the pantograph voltage, reflecting troubled convergence of the non-linear calculation. Fig. 7 superimposes the same curves for the 8 trains and shows that the same phenomena happen for all the trains. Hence, a deeper analysis is needed in order to check the correctness of the simulation and the feasibility of the system.

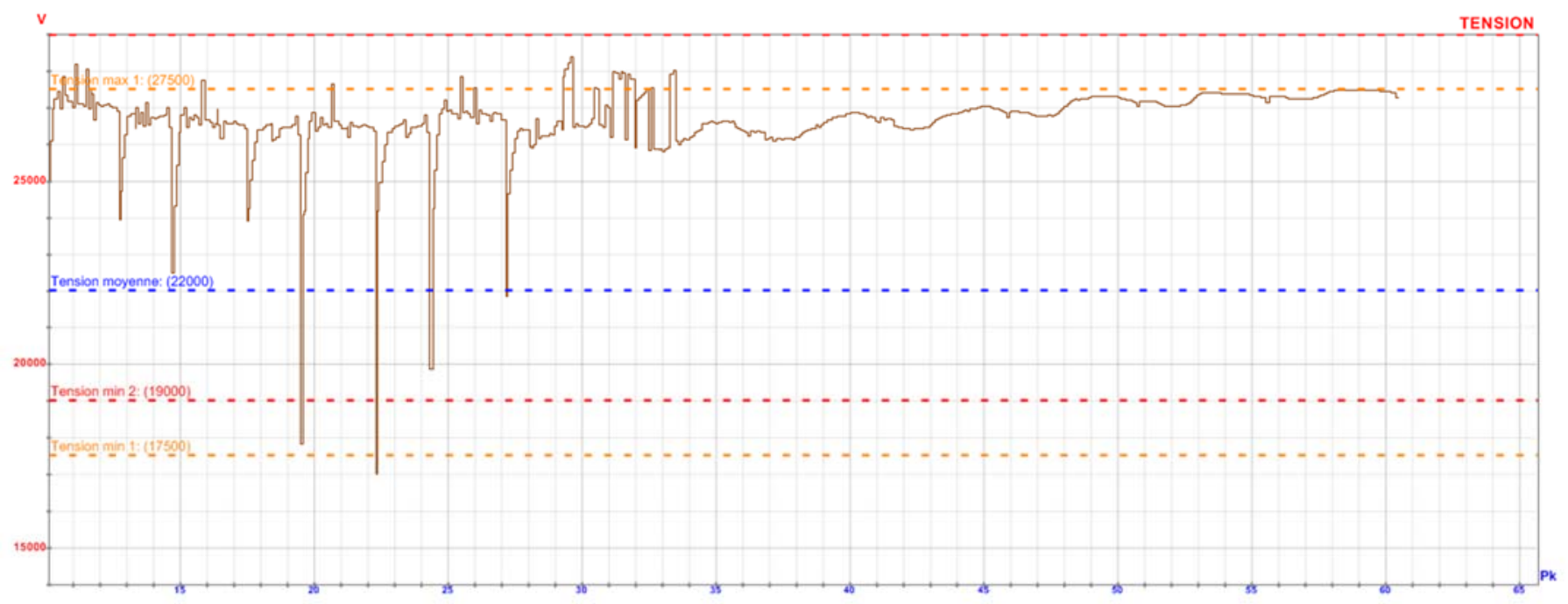

Fig. 6 : Line voltage versus train position, for one of the eight running trains 


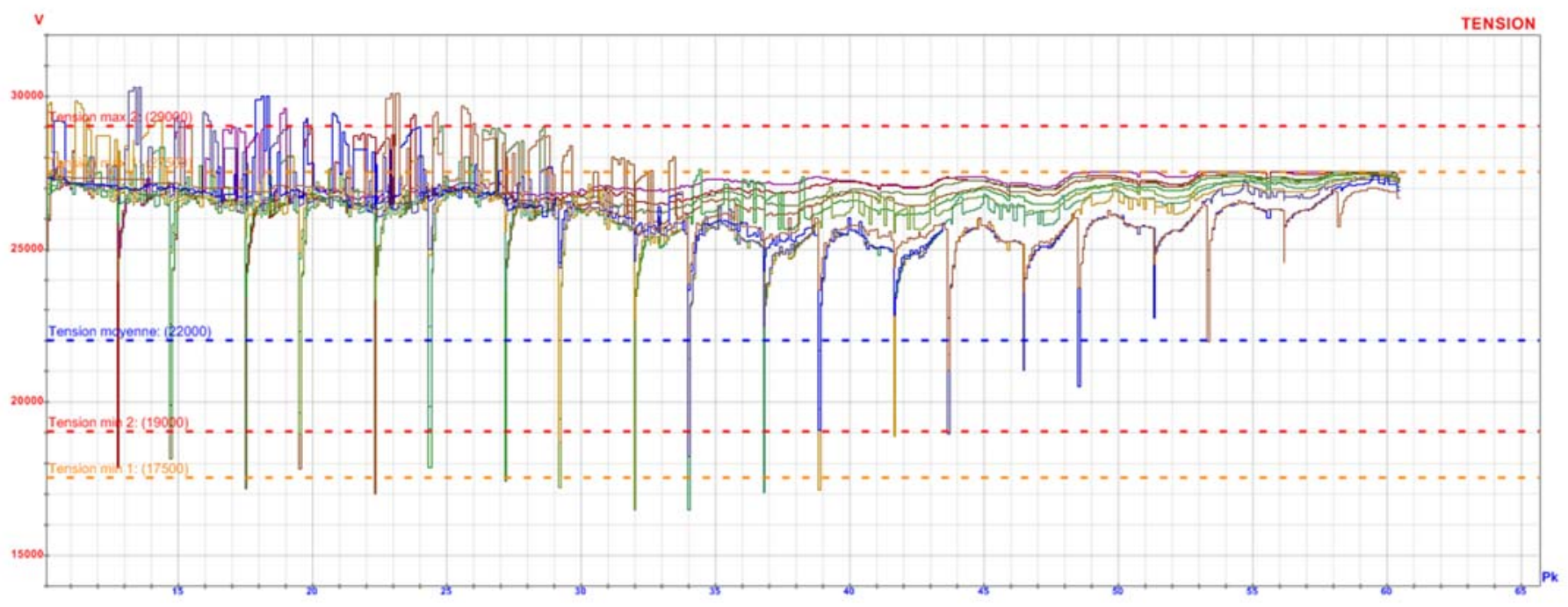

Fig. 7 : Line voltage versus train position, for each of the eight running trains

This problem is very difficult to assess purely in terms of voltage and requires to assess the admissible maximum power of the line with additional complexity because of the capacitance. The results can be interpreted and understood by analyzing the problem for a specific position of the trains, for which convergence is difficult or fails. To do this, we have "frozen" the system at a given time step, for given trains positions and powers, and we have simplified it for easier interpretation. The equivalent electrical circuit was modeled using the modified nodal method [10][11], which allows accounting for voltage constraints: $27.25 \mathrm{kV}$ at the SVC terminals in the present case. The method results in a non linear problem, whose unknown are the voltage at the different nodes and the currents in branches where a voltage constraint applies (SVC current in our case, $90^{\circ}$ ahead of the voltage). The trains were modeled as given power loads, which also contributes to the non linear behavior of the system. The non linear matrix system was solved using Newton method, analytical formula of the derivatives and a convergence criterion set to $10^{-16}$ (double precision calculation in scilab environment). As underlined in section II.B and shown by Fig.4, two voltage solutions exist for a load with given active power. This requires a careful choice of the starting point of the non linear solver, in order to follow the upper branch of the voltage-power curve. Otherwise, the calculus may converge toward the lower branch, which is not desirable.

Assessing the maximum load of the line is not an easy task when dealing with multiple loads: one could add the loads one after another, but this leads to a large number of possible combinations. Instead, we have chosen to progressively increase the power of all the loads, from 0 to $100 \%$ of their final value by step of $10 \%$.

Fig. 8 shows the resulting line voltages: the feeding substation is at the left end of the curve, whereas the SVC is at the right end. Each open circle in-between represents a train and the last one of them is very close to the SVC. As one moves away from the substation, the line voltage first decreases and then increases as one gets closer to the SVC.
The voltage constraint at the end of the line is clearly seen. The blue curves correspond to loads ranging between $10 \%$ and $60 \%$ of the final load and for which convergence was reached with the desired precision. In each case, the voltage constraint at the SVC terminals is strictly respected. The red curves corresponds to loads between $70 \%$ and $100 \%$, for which convergence could not be obtained with the desired precision. Fig. 9 plots the normed residual of the non linear problem versus the load. It shows that the residual is zero (actually around $10^{-16}$ ) for loads up to $60 \%$ of the final one, and then increases as the load exceeds the maximal possible value: the calculation gives the closest solution to the problem, but this solution is not physically acceptable, although the overall shape seems correct.

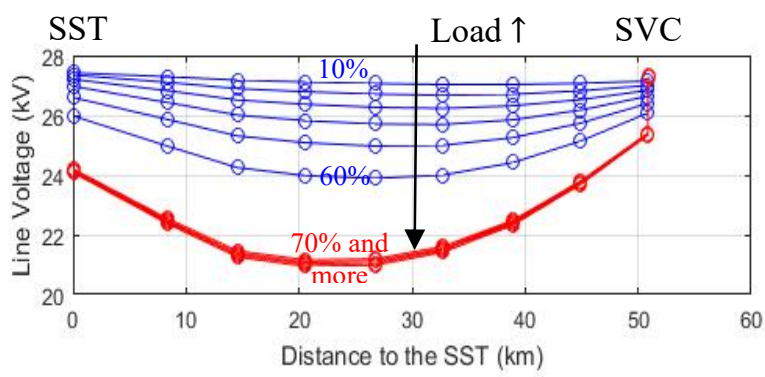

Fig. 8 : Line voltage. The convergence error is plotted in Fig.9.

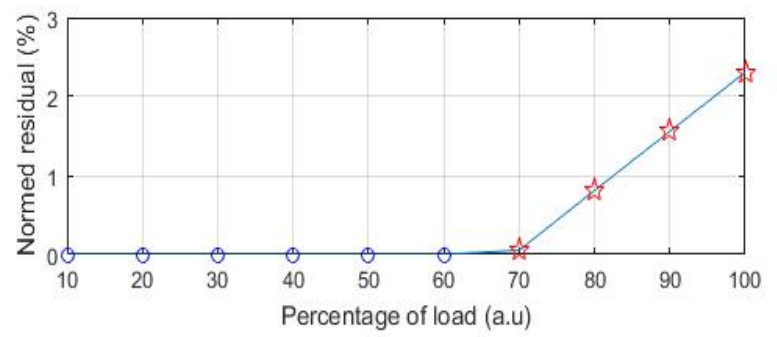

Fig. 9 : Normed residual for an increasing load. 
Another indicator of this inconsistency is the fact that the voltage constraint at the SVC terminals is no longer strictly respected, with a discrepancy representative of the convergence error. These results allow a better interpretation of the curves presented in Fig. 7. If the line load exceeds its transmissible value, convergence fails whatever the SVC capacity, but a convergence criterion not severe enough fails to detect this unphysical situation. It is common to use a convergence criterion around $1 \%$. This value is acceptable in usual conditions, but not any more when the system operates close to its physical limits: in the present case this may lead to false results with no warning. The voltage drops on some intervals can be interpreted as convergence toward the lower part of the voltage-power curve. This solution exists but is not the one which corresponds to the real system. Hence, the numerical implementation of the non linear calculation must be done carefully, so as to avoid this artefact.

\section{ACKNOWLEDGMENT}

E.Sourdille thanks his colleagues at the SNCF Réseau Electrical Traction department and in particular $\mathrm{Mr}$ Thibaut Aubry. Also many thanks to Mr Eric Guillaume who enabled to better interpret the parabola and helped establishing the formulation.

\section{REFERENCES}

[1] Mao Baohua, Jia Wenzheng, Chen Shaokuan, Liu Jianfeg, "Computeraided multi-train simulator for rail traffic," IEEE Vehicular Electronics and Safety, 13-15 December 2007.

[2] T.K. Ho, B.H. Mao, Z.Z. Yuan, H.D. Liu, Y.F. Fung, "Computer simulation and modeling in railway applications," Computer Physics Communications 143, 2002.

[3] Chang Han Bae, "A simulation study of installation locations and capacity of regeneratuve absorption in DC $1500 \mathrm{~V}$ electric railways system," Simulation Modelling Pratice and Theory 17, 2009, pp. 829838.

[4] A. Finlayson, C.J. Goodman, R.D. White, "Investigation into the computational techniques of power system modelling for a DC railway,"

\section{CONCLUSION}

In specific conditions, although it is not desirable, the railway power feeding system has to be operated close to its physical limits. These limits have to be well understood and accounted for in simulation tools. We have presented an approach based on the use of the convergence criteria to characterize this phenomenon. However, this indicator is used afterwards to check the validity of the simulation in a trial and error sizing approach. Further work should rely on the limiting parabola of the system and a simplified representation of the system to establish a sizing criteria before simulating the system.

Computers in railways X, WIT Transactions on The built Environment, Vol 88, 2006.

[5] E. Guillaume, "Dimensionnement économique d'une ligne 1500 V DC," SNCF internal report, March 2014, unpublished.

[6] E. Guillaume, "Formulaire du survolteur," SNCF internal report, March 2012, unpublished.

[7] EN 50388:2012, "Railway Applications. Power supply and rolling stock. Technical criteria for the coordination between power supply (substation) and rolling stock to achieve interoperability ," CENELEC, 2012.

[8] EN 50163:2005, "Railway Applications - Supply voltages of traction system ," CENELEC, 2005.

[9] Marcel Tessier, "Traction Electrique et Thermo Electrique", Edition Scientifique Riber, 1978

[10] Chung-Wen Ho, Albert E. Ruehli, Pierce A. Brennank, "The modified nodal approach to network analysis," IEEE Transactions on circuits and systems, vol cas-22, no. 6, June 1975.

[11] B. Desjouis, G. Remy, F. Ossart, J . Bigeon, E.Sourdille, "A new generic formulation dedicated to electrified railway systems », 2015 Intern. Conf. on Electrical Systems for Aircraft, Railway, Ship Propulsion and Road Vehicles - doi : 10.1109/ESARS.2015.7101437 